\title{
Radiative Lifetimes of the Bound Excited States of $\mathrm{Pt}^{-}$
}

\author{
KC Chartkunchand, ${ }^{1, *}$ M. Kamińska, ${ }^{1,2}$ E. K. Anderson, ${ }^{1}$ M. K. Kristiansson, ${ }^{3}$ \\ G. Eklund, ${ }^{1}$ O. M. Hole,${ }^{1}$ R. F. Nascimento,${ }^{1,4}$ M. Blom, ${ }^{1}$ M. Björkhage, ${ }^{1}$ \\ A. Källberg, ${ }^{1}$ P. Löfgren, ${ }^{1}$ P. Reinhed, ${ }^{1}$ S. Rosén, ${ }^{1}$ A. Simonsson,${ }^{1}$ R. D. \\ Thomas, ${ }^{1}$ S. Mannervik, ${ }^{1}$ V. T. Davis,${ }^{5}$ P. A. Neill, ${ }^{5}$ J. S. Thompson ${ }^{5}$ \\ D. Hanstorp ${ }^{3}$ H. Zettergren, ${ }^{1}$ H. Cederquist, ${ }^{1}$ and H. T. Schmidt ${ }^{1},+$ \\ ${ }^{1}$ Department of Physics, Stockholm University, \\ AlbaNova, SE-106 91 Stockholm, Sweden \\ ${ }^{2}$ Institute of Physics, Jan Kochanowski University, 25-369 Kielce, Poland \\ ${ }^{3}$ Department of Physics, University of Gothenburg, SE-412 96 Gothenburg, Sweden \\ ${ }^{4}$ Centro Federal de Educação Tecnológica Celso \\ Suckow da Fonseca, Petrópolis, 25620-003, RJ, Brazil \\ ${ }^{5}$ Department of Physics, University of Nevada, \\ Reno, Nevada 89557, United States
}

(Dated: November 9, 2018)

\begin{abstract}
The intrinsic radiative lifetimes of the $5 d^{10} 6 s{ }^{2} \mathrm{~S}_{1 / 2}$ and $5 d^{9} 6 s^{2}{ }^{2} \mathrm{D}_{3 / 2}$ bound excited states in the platinum anion $\mathrm{Pt}^{-}$have been studied at cryogenic temperatures at the DESIREE (Double ElectroStatic Ion Ring Experiment) facility at Stockholm University. The intrinsic lifetime of the higher-lying $5 d^{10} 6 s{ }^{2} \mathrm{~S}_{1 / 2}$ state was measured to be $2.54 \pm 0.10 \mathrm{~s}$, while only a lifetime in the range of 50-200 ms could be estimated for the $5 d^{9} 6 s^{2}{ }^{2} \mathrm{D}_{3 / 2}$ fine-structure level. The storage lifetime of the $\mathrm{Pt}^{-}$ion beam was measured to be a little over 15 minutes at a ring temperature of $13 \mathrm{~K}$. The present study is the first to report the lifetime of an atomic negative ion in an excited bound state with an electron configuration different from that of the ground state.
\end{abstract}

PACS numbers: $32.70 . \mathrm{Cs}, 32.80 . \mathrm{Gc}, 07.20 . \mathrm{Mc}$

\footnotetext{
* Corresponding author: kiattichart.chartkunchand@fysik.su.se

$\dagger$ schmidt@fysik.su.se
} 


\section{INTRODUCTION}

Negative ions are fascinating quantum systems that illustrate the subtle nature of electronic structure and dynamics on the atomic scale. Due to the short range of the forces that hold these systems together, the number of bound quantum states is strongly limited. Some atoms do not even form stable anions, or have only a single bound state [1, 2]. Other atomic anions, however, possess one or a few bound excited states. These include higher-lying $L S$ terms or fine-structure levels of the same electron configuration as the anionic ground state [3, 4] and/or bound excited states with different electron configurations [5]. In most cases, the ground and excited atomic anionic states have the same parity. Single-photon electric dipole (E1) transitions are then forbidden by the parity selection rule and the corresponding excited states are often very long-lived. This makes studies based on conventional optical spectroscopy methods difficult and the lifetimes of bound excited states in atomic anions are, with few exceptions, unknown. From a theoretical perspective, studies of negative ions are made more challenging by the need to accurately describe electron correlation effects, which are much more important for anions than for neutrals or cations. Measurements of negative ion properties such as binding energies and lifetimes can thus provide critical benchmarks for theoretical methods treating such correlation effects in atomic systems in general.

The characterization of atomic and molecular anions is crucial for several fields of science, as well as for certain technological applications. Negative ions are, for example, believed to play key roles as absorbers, emitters, and reaction partners in stellar and planetary atmospheres, and in the interstellar medium [6 12]. Additionally, the ability to produce beams of atomic anions forms the basis for a range of powerful and widely-used radiological dating techniques [4, 13]. Furthermore, the discovery of bound excited states in $\mathrm{La}^{-}$[14], $\mathrm{Ce}^{-}$[15], and $\mathrm{Os}^{-}[16,17]$ with parities opposite to those of the corresponding ground states has opened up the possibility of laser cooling of these atomic anions. Such cold anions could be used to sympathetically cool anti-protons to facilitate the production of ultra-cold anti-hydrogen for use in high-precision spectroscopic tests of CPT symmetry, and for measurements of the effects of gravity on anti-matter [18]. In each of the above examples, a detailed understanding of anion properties is absolutely necessary.

Only recently have techniques for long-term storage of negative ions (seconds, minutes, 
and beyond) become available, allowing for studies of long-lived anionic excited bound states [19 31]. The cryogenic ion storage rings DESIREE [29, 32, 33], CSR [30], and RICE [34], which can be used to store keV ion beams for up to hours in cryogenic, magneticfield-free environments, are at the forefront in this field [35]. Some of the capabilities of the DESIREE facility were recently demonstrated through measurements of the radiative lifetimes of the long-lived, excited fine-structure level in the $n p^{5}{ }^{2} \mathrm{P}_{1 / 2} \rightarrow n p^{5}{ }^{2} \mathrm{P}_{3 / 2}$ transition in $\mathrm{S}^{-}(n=3, \tau=503 \pm 54 \mathrm{~s}[33]), \mathrm{Se}^{-}(n=4, \tau=4.78 \pm 0.18 \mathrm{~s}$ [33] $)$, and $\mathrm{Te}^{-}$ $(n=5, \tau=0.463 \pm 0.008 \mathrm{~s}[33])$, and of the $3 d^{9} 4 s^{2}{ }^{2} \mathrm{D}_{3 / 2} \rightarrow 3 d^{9} 4 s^{2}{ }^{2} \mathrm{D}_{5 / 2}$ transition in $\mathrm{Ni}^{-}$ $(\tau=15.1 \pm 0.4 \mathrm{~s})[36]$.

The electronic structure of the platinum anion, $\mathrm{Pt}^{-}$, has been studied previously and its energy level diagram is shown in Fig. 1. The electron affinity of Pt was measured to be $2.12510(5) \mathrm{eV}$ by means of the laser photodetachment threshold (LPT) technique [37]. Two-photon detachment spectroscopy was utilized to determine the excitation energy of the $5 d^{9} 6 s^{2}{ }^{2} \mathrm{D}_{3 / 2}$ fine-structure level, which was found to lie $1.20775(6) \mathrm{eV}$ above the $5 d^{9} 6 s^{2}$ ${ }^{2} \mathrm{D}_{5 / 2}$ anionic ground state [38]. The highest-lying $5 d^{10} 6 s{ }^{2} \mathrm{~S}_{1 / 2} \mathrm{Pt}^{-}$bound state, which has a different electron configuration from that of the ground state, was measured to lie $1.27567(161) \mathrm{eV}$ above the $\mathrm{Pt}^{-}$ground state [39]. Both excited states are expected to be long-lived since E1 transitions to the ground state are forbidden by the parity selection rule. The $5 d^{9} 6 s^{2}{ }^{2} \mathrm{D}_{3 / 2}$ fine-structure level may decay by an M1 transition to the ground state, while decay from the $5 d^{10} 6 s{ }^{2} \mathrm{~S}_{1 / 2}$ state may proceed through E2 transitions to the $5 d^{9} 6 s^{2}$ ${ }^{2} \mathrm{D}_{5 / 2,3 / 2}$ levels.

Thøgersen et al. [38] used multi-configurational Dirac-Fock methods to calculate a spontaneous decay rate of $\Gamma=14 \mathrm{~s}^{-1}$ for the $5 d^{9} 6 s^{2}{ }^{2} \mathrm{D}_{3 / 2} \rightarrow 5 d^{9} 6 s^{2}{ }^{2} \mathrm{D}_{5 / 2}$ transition in $\mathrm{Pt}^{-}$, corresponding to a lifetime of $\tau=71 \mathrm{~ms}$. It is interesting to note that a scaling of the $\tau=15.1 \pm 0.4 \mathrm{~s}$ experimental lifetime of the $3 d^{9} 4 s^{2}{ }^{2} \mathrm{D}_{3 / 2}$ level in $\mathrm{Ni}^{-}[36]$ with the third power of the ratio between the corresponding transition energies $\mathrm{in} \mathrm{Ni}^{-}$and $\mathrm{Pt}^{-}$suggests a lifetime of the $5 d^{9} 6 s^{2}{ }^{2} \mathrm{D}_{3 / 2}$ level in $\mathrm{Pt}^{-}$of $\tau=53 \pm 2 \mathrm{~ms}$ [40]. To the best of our knowledge, no calculation of the $5 d^{10} 6 s{ }^{2} \mathrm{~S}_{1 / 2} \rightarrow 5 d^{9} 6 s^{2}{ }^{2} \mathrm{D}_{3 / 2,5 / 2}$ transition rates in $\mathrm{Pt}^{-}$, which are the main quantities of interest in the present study, has been reported in the literature. 


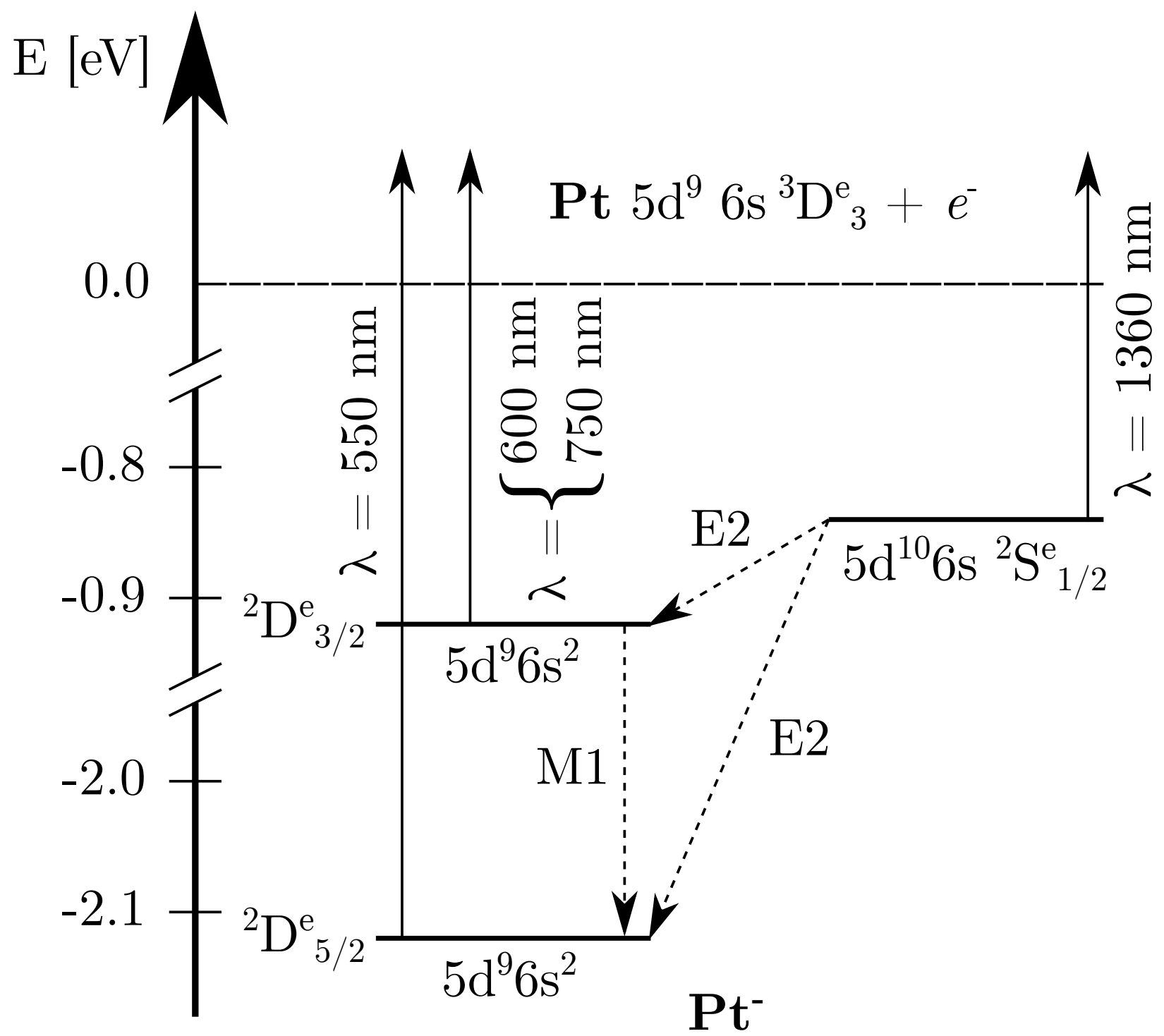

FIG. 1. Energy-level diagram of the three bound states of $\mathrm{Pt}^{-}$. The vertical scale indicates total energies with respect to the ground state of the neutral Pt atom. Magnetic dipole (M1) and electric quadrupole (E2) transitions are the lowest-order transitions possible between the ground and excited states of $\mathrm{Pt}^{-}$.

\section{EXPERIMENTAL APPARATUS}

The present experiment was conducted at the cryogenic electrostatic ion beam storage ring DESIREE. A full description of this facility is provided in Refs. [29, 32], so only a brief summary is given here. Platinum anions $\left(\mathrm{Pt}^{-}\right)$were produced in a SNICS II cesium-sputter ion source [41]. The $\mathrm{Pt}^{-}$ions were accelerated to an energy of $10 \mathrm{keV}$ and passed through a 


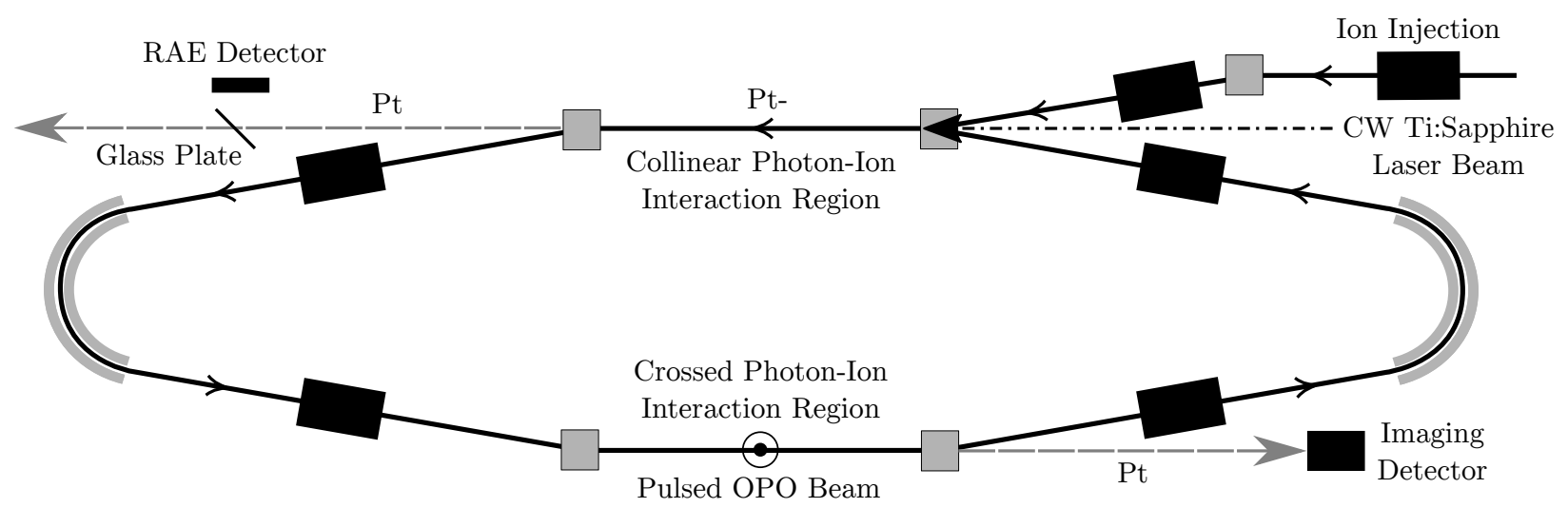

FIG. 2. Schematic of one of the ion storage rings of DESIREE. $10 \mathrm{keV}$ beams of $\mathrm{Pt}^{-}$are injected and stored in the ring. The output of a continuous wave $(\mathrm{CW})$ Ti:Sapphire laser propagates along the ion beam direction in one of the straight sections of the ring and can be overlapped with the stored ions in a collinear geometry, while the output of a pulsed optical parametric oscillator (OPO) can be crossed with the ion beam at $90^{\circ}$ in the other straight section. Neutral Pt atoms produced through photodetachment in the collinear interaction region are detected by a resistive anode encoder (RAE) detector, while those produced in the crossed interaction region are detected by an imaging detector.

$90^{\circ}$ analyzing magnet for mass selection before injection into the symmetric ion storage ring of DESIREE [29, 32], as shown in Fig. 2. A typical mass scan of the $\mathrm{Pt}^{-}$beam is shown in Fig. 3. The ratios of ion beam currents produced for each $\mathrm{Pt}^{-}$mass agreed well with the natural abundances of $\mathrm{Pt}$ isotopes, indicating that only small amounts of $\mathrm{PtH}^{-}$were produced in the ion source. For the present experiment, the ${ }^{194} \mathrm{Pt}^{-}$isotope was selected to minimize the risk of contamination by other anionic species at masses 195, 196 and $198 \mathrm{u}$. Such contaminations were observed in earlier experiments utilizing a similar type of ion source [39]. As examples, the ${ }^{194,195} \mathrm{PtH}^{-}$and ${ }^{63,65} \mathrm{Cu}^{133} \mathrm{Cs}^{-}$molecular anions overlap some $\mathrm{Pt}^{-}$masses above $194 \mathrm{u}$.

Two different photon sources and interaction geometries were used for probing the populations of the three bound states of the $\mathrm{Pt}^{-}$ions as functions of time after injection in the ion storage ring. A continuous wave (CW) Sirah Matisse Ti:Sapphire laser was used in a collinear interaction geometry in one of the straight sections of the ion storage ring as shown in Fig. 2. The CW laser beam was propagated along the ion beam direction, allowing for a large photon-ion interaction volume. Neutral Pt atoms produced by photodetachment 


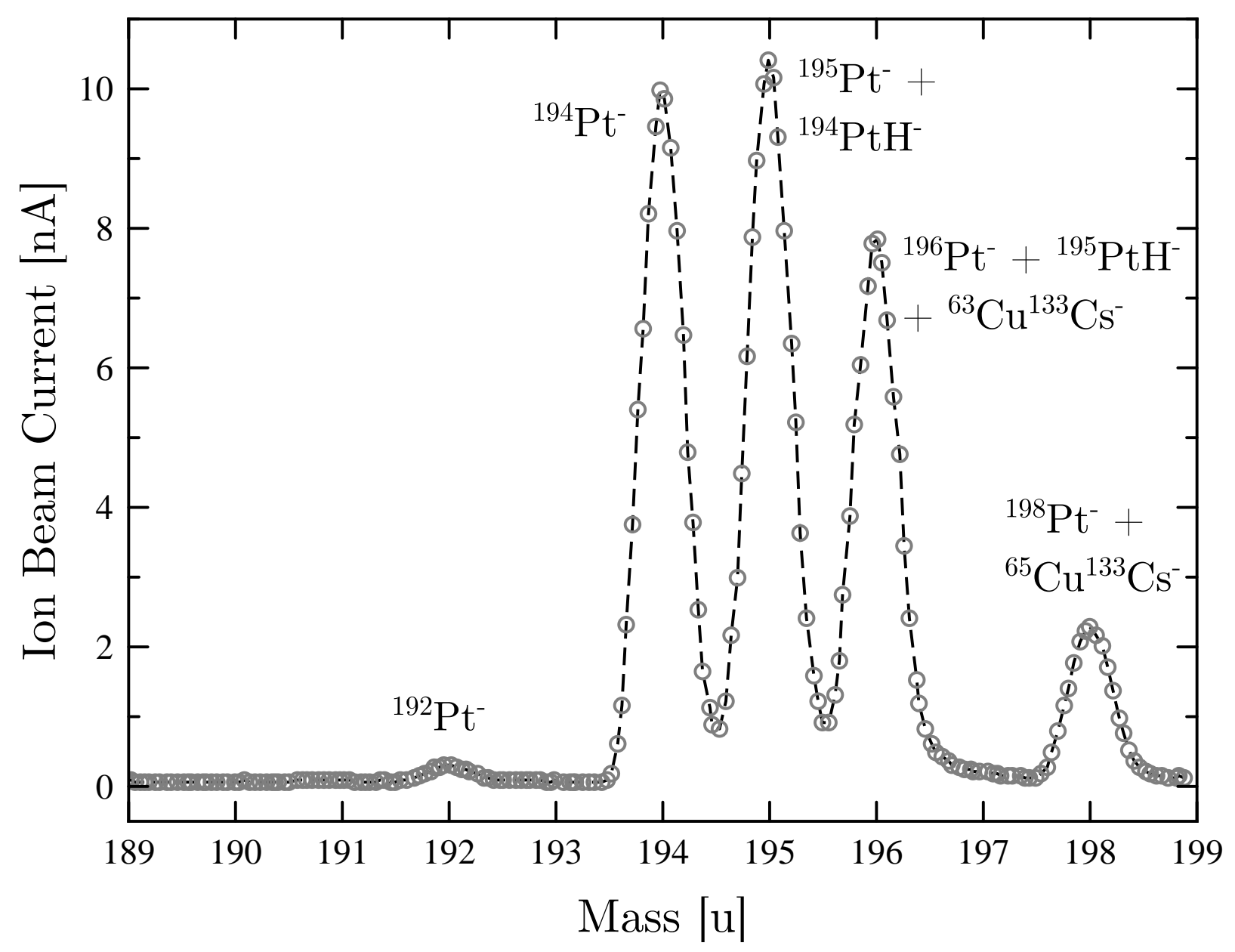

FIG. 3. Mass scan of $\mathrm{Pt}^{-}$ion beam in the range of $190-199 \mathrm{u}$. The ${ }^{194} \mathrm{Pt}^{-}$isotope was used throughout the experiment to avoid contamination from other anionic species that share the same mass as the 195, 196 and $198 \mathrm{u}$ isotopes of $\mathrm{Pt}$.

leave the ring and strike a gold-coated glass plate, producing secondary electrons that are registered by a particle detector consisting of three microchannel plates (MCPs) and a resistive anode encoder (RAE) [29, 32, 42]. Alternatively, the output of an Ekspla NT300-series tunable pulsed optical parametric oscillator $(\mathrm{OPO})$, with a pulse width of $\sim 5 \mathrm{~ns}$ and repetition rate of $10 \mathrm{~Hz}$, was used in a crossed-beam interaction geometry in the opposite straight section of the ring (see Fig. 2). In this case, neutral Pt atoms are counted by an imaging detector, consisting of a triple-stack MCP with a phosphor screen anode viewed by a photomultiplier tube [29, 32, 42]. In both cases, background signals were mainly due to detector dark counts and only to a limited extent to neutral Pt atoms produced through collisions with the very dilute residual gas of $10^{3}-10^{4} \mathrm{H}_{2}$ molecules per $\mathrm{cm}^{3}$ [32]. 
The lifetimes of the bound states of $\mathrm{Pt}^{-}$were measured by state-selective probing of their populations as functions of time after ion injection using either the OPO or the Ti:Sapphire laser. The pulsed OPO offered a broader wavelength tunability (200-2600 nm) compared to the Ti:Sapphire laser $(700-990 \mathrm{~nm})$, allowing for detachment from only the $5 d^{10} 6 s{ }^{2} \mathrm{~S}_{1 / 2}$ excited state with $1360 \mathrm{~nm}$ light $(0.912 \mathrm{eV}$ photon energy), detachment from both excited states with $600 \mathrm{~nm}$ light $(2.07 \mathrm{eV}$ photon energy), and detachment from all three bound states with $550 \mathrm{~nm}$ light $(2.25 \mathrm{eV}$ photon energy). The short pulse length of the OPO also allowed for efficient background suppression by only counting Pt atoms on the imaging detector within a $10 \mu$ s-wide time window around the OPO pulse. The OPO pulse energy and ion beam current were adjusted such that the probability for a given OPO pulse to produce one $\mathrm{Pt}$ atom was $<0.01$. This was done in order to limit the probability that two neutrals were produced by a single OPO pulse, which would be counted as only one event by the detector due to its finite response time (resulting in a so-called "counting saturation"). Background contributions from collisions in the residual gas and detector dark counts were measured by counting events within a 40 ms-wide time window between the OPO pulses.

The CW Ti:Sapphire laser could produce a higher time-averaged neutral signal rate via photodetachment compared to the pulsed OPO. This was due to a combination of the much larger ion-photon interaction volume provided by the collinear interaction geometry and the use of higher time-averaged photon intensities and ion beam currents since "counting saturation" was no longer an issue with the CW laser. The CW mode of the Ti:Sapphire laser was also better suited for studying lifetimes shorter than $100 \mathrm{~ms}$, a situation in which the fixed $10 \mathrm{~Hz}$ repetition rate of the OPO became a limiting factor. The Ti:Sapphire laser was used at a wavelength of $750 \mathrm{~nm}(1.65 \mathrm{eV}$ photon energy), allowing for photodetachment from both excited states of $\mathrm{Pt}^{-}$.

\section{RESULTS AND DISCUSSION}

In order to extract effective lifetimes from the measured decay curves, fitting functions in the form of exponentials or sums of exponentials were used [36]. A weighted, non-linear least-squares routine was used in fitting the data, with each data point weighted according to its statistical uncertainty assuming a Poisson distribution. Each fit resulted in the effective

lifetime of each $\mathrm{Pt}^{-}$state, denoted by $\tau_{5 / 2}^{\text {eff }}, \tau_{3 / 2}^{\text {eff }}$, and $\tau_{1 / 2}^{\text {eff }}$ for the $5 d^{9} 6 s^{2}{ }^{2} \mathrm{D}_{5 / 2}, 5 d^{9} 6 s^{2}{ }^{2} \mathrm{D}_{3 / 2}$, 
and $5 d^{10} 6 s{ }^{2} \mathrm{~S}_{1 / 2}$ states, respectively. The effective lifetime of the anionic ground state, $\tau_{5 / 2}^{\text {eff }}$, corresponds to the storage lifetime of the $\mathrm{Pt}^{-}$ions in the ring. Photon intensities used to probe the ions were adjusted such that photodetachment rates remained negligible in relation to the effective decay rates to be measured. The effective decay rate of a bound excited state in the stored ion beam is then the sum of the corresponding intrinsic decay rate and the rate at which the excited ions are lost through collisional detachment with the residual $\mathrm{H}_{2}$ gas or through ion storage imperfections (e.g., voltage ripples on the ion optical elements in the ring) [32, 33].

\section{A. The $5 d^{10} 6 s{ }^{2} \mathbf{S}_{1 / 2} \rightarrow 5 d^{9} 6 s^{2}{ }^{2} \mathbf{D}_{5 / 2}$ Transition}

The measured decay curve for the $5 d^{10} 6 s{ }^{2} \mathrm{~S}_{1 / 2}$ state is shown in the upper plot of Fig. 4 , along with the decay curve from which the ion beam storage time is determined (lower plot). The effective lifetime for the $5 d^{10} 6 s{ }^{2} \mathrm{~S}_{1 / 2}$ state was found to be $\tau_{1 / 2}^{\text {eff }}=2.53 \pm 0.08 \mathrm{~s}$, while the effective ion storage lifetime was found to be $\tau_{5 / 2}^{\text {eff }}=946 \pm 25 \mathrm{~s}$. The latter lifetime yields an ion beam decay rate of $\Gamma_{5 / 2}^{\mathrm{eff}}=1 / \tau_{5 / 2}^{\mathrm{eff}}=0.00106 \pm 0.00003 \mathrm{~s}^{-1}$. This value can be used to deduce the intrinsic lifetime $\tau_{1 / 2}^{\text {int }}$ of the excited ${ }^{2} \mathrm{~S}_{1 / 2}$ state from the corresponding effective lifetime. The intrinsic lifetime is determined by applying a small correction to $\tau_{1 / 2}^{\text {eff }}$ that accounts for the fact that $\mathrm{Pt}^{-}$ions in the excited state may be lost in collisions with the residual gas or by imperfections in the ion storage condition. This is expressed by $1 / \tau_{1 / 2}^{\text {int }}=1 / \tau_{1 / 2}^{\text {eff }}-[\beta+\eta(1-\beta)] \times \Gamma_{5 / 2}^{\text {eff }}$, in which $\beta$ is the fraction of losses due to storage imperfections and $\eta$ is the ratio between the cross-sections for collisional detachment in $\mathrm{H}_{2}$ (which completely dominates the residual gas at $13 \mathrm{~K} \mathrm{[32])} \mathrm{of} \mathrm{Pt}^{-}$in the excited and ground states. By assuming $\beta+\eta(1-\beta)=1 \pm 10$, an intrinsic lifetime for the $5 d^{10} 6 s{ }^{2} \mathrm{~S}_{1 / 2}$ state of $\tau_{1 / 2}^{\text {int }}=2.54 \pm 0.10 \mathrm{~s}$ is determined. This is an extremely conservative assumption; in other cases, $\eta$ has been measured to be close to one [33, 36].

The ${ }^{2} \mathrm{~S}_{1 / 2}$ state is expected to decay to the anionic ground state and the $5 d^{9} 6 s^{2}{ }^{2} \mathrm{D}_{3 / 2}$ excited fine-structure level via E2 transitions (see Fig. 1). An estimate of the ratio between the E2 decay rates can be made by noting that the E2 spontaneous transition rate is proportional to the square of the matrix element of the electric quadrupole operator between the ${ }^{2} \mathrm{~S}_{1 / 2}$ and ${ }^{2} \mathrm{D}_{5 / 2,3 / 2}$ states and the fifth power of the energy separation between the ${ }^{2} \mathrm{~S}$ and ${ }^{2} \mathrm{D}$ states (see, e.g., [43]). Assuming that the matrix elements between the ${ }^{2} \mathrm{~S}_{1 / 2}$ state 


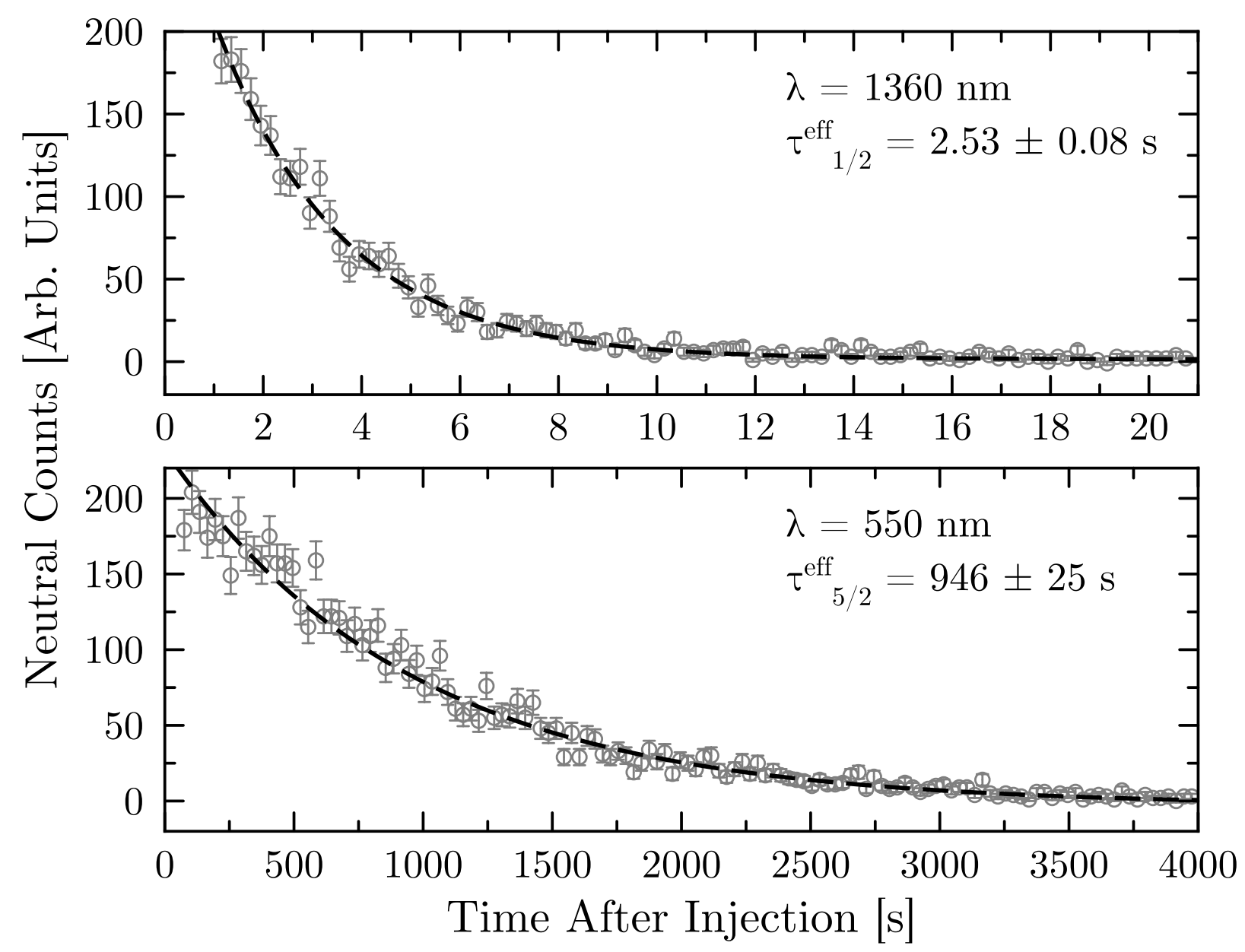

FIG. 4. Decay curves of the $\mathrm{Pt}^{-}$beam as a function of time at the base storage ring temperature of $13 \mathrm{~K}$. The upper plot shows decay signal from only the $5 d^{10} 6 s{ }^{2} \mathrm{~S}_{1 / 2}$ state, while the lower plot contains contributions from all three states of $\mathrm{Pt}^{-}$. Note the difference in time scales between the upper and lower plots.

and the two $5 d^{9} 6 s^{2}{ }^{2} \mathrm{D}_{5 / 2,3 / 2}$ states are of a similar magnitude, and using the experimentallydetermined energy separations [38, 39], the transition to the ${ }^{2} \mathrm{D}_{3 / 2}$ level can be estimated to be seven orders-of-magnitude weaker than the transition to the ${ }^{2} \mathrm{D}_{5 / 2}$ ground state. The $5 d^{10} 6 s{ }^{2} \mathrm{~S}_{1 / 2} \rightarrow 5 d^{9} 6 s^{2}{ }^{2} \mathrm{D}_{3 / 2}$ decay rate is thus estimated to be in the $10^{-8} \mathrm{~s}^{-1}$ range by using the $2.54 \pm 0.10 \mathrm{~s}$ lifetime of the upper state. 


\section{B. The $5 d^{9} 6 s^{2}{ }^{2} \mathbf{D}_{3 / 2} \rightarrow 5 d^{9} 6 s^{2}{ }^{2} \mathbf{D}_{5 / 2}$ Transition}

For the $5 d^{9} 6 s^{2}{ }^{2} \mathrm{D}_{3 / 2}$ excited fine-structure level of $\mathrm{Pt}^{-}$, exploratory measurements were first carried out at an OPO wavelength of $600 \mathrm{~nm}$ over a $20 \mathrm{~s}$ measurement window, i.e., the populations in this state and in the higher-lying ${ }^{2} \mathrm{~S}_{1 / 2}$ state were probed for times up to $20 \mathrm{~s}$ after injection of ions into the ring. Analysis of the measured decay curve revealed a decay time on the order of the effective lifetime of the $5 d^{10} 6 s{ }^{2} \mathrm{~S}_{1 / 2}$ excited state, with no indication of any other components that may be due to decay from the $5 d^{9} 6 s^{2}{ }^{2} \mathrm{D}_{3 / 2}$ level. There are two possibilities regarding this state: either its effective lifetime is very similar to that of the higher-lying $5 d^{10} 6 s{ }^{2} \mathrm{~S}_{1 / 2}$ state, or it decays on a much shorter time scale and is thus more difficult to detect due to the "background" from the decay of the higher-lying state. In light of the theoretical prediction for the lifetime of the $5 d^{9} 6 s^{2}{ }^{2} \mathrm{D}_{3 / 2}$ level of $71 \mathrm{~ms}$ [38], the possibility for a short ${ }^{2} \mathrm{D}_{3 / 2}$ lifetime was investigated using the $\mathrm{CW}$ Ti:Sapphire laser at a wavelength of $750 \mathrm{~nm}$ in the collinear interaction geometry (see Fig. 2). Measurements of decay curves were performed over $1 \mathrm{~s}$ and $15 \mathrm{~s}$ measurement windows, using $\sim 3 \mathrm{nA}$ of $\mathrm{Pt}^{-}$ion beam current in both cases. The measurement with the $15 \mathrm{~s}$ time window revealed an effective lifetime for the excited states around $2.6 \mathrm{~s}$, which is in agreement with the effective lifetime of the $5 d^{10} 6 s{ }^{2} \mathrm{~S}_{1 / 2}$ state as measured with the OPO. Results from the measurement with the $1 \mathrm{~s}$ time window are shown in Fig. 5 . In order to test for the presence of any short-lived component in the decay curve, the exponential function used to fit the $15 \mathrm{~s}$ measurement window data was subtracted from the $1 \mathrm{~s}$ data, thereby removing contributions from the longer-lived ${ }^{2} \mathrm{~S}_{1 / 2}$ excited state. This procedure revealed the presence of a short-lived signal which disappears $\sim 100$ ms after injection, which may be due to decay of the $5 d^{9} 6 s^{2}{ }^{2} \mathrm{D}_{3 / 2}$ excited state. Attempts at a more direct measurement of the shortlived signal by increasing the ion beam current $(>10 \mathrm{nA})$ to yield a larger overall signal rate were not fully conclusive. This was attributed to various ion-ion effects such as intra-beam scattering, whose relaxation times could be similar to the times in which decay from the $5 d^{9} 6 s^{2}{ }^{2} \mathrm{D}_{3 / 2}$ state would be observed. Due to these difficulties, only a range of 50-200 ms could be estimated for the lifetime of the $5 d^{9} 6 s^{2}{ }^{2} \mathrm{D}_{3 / 2}$ bound excited state. 


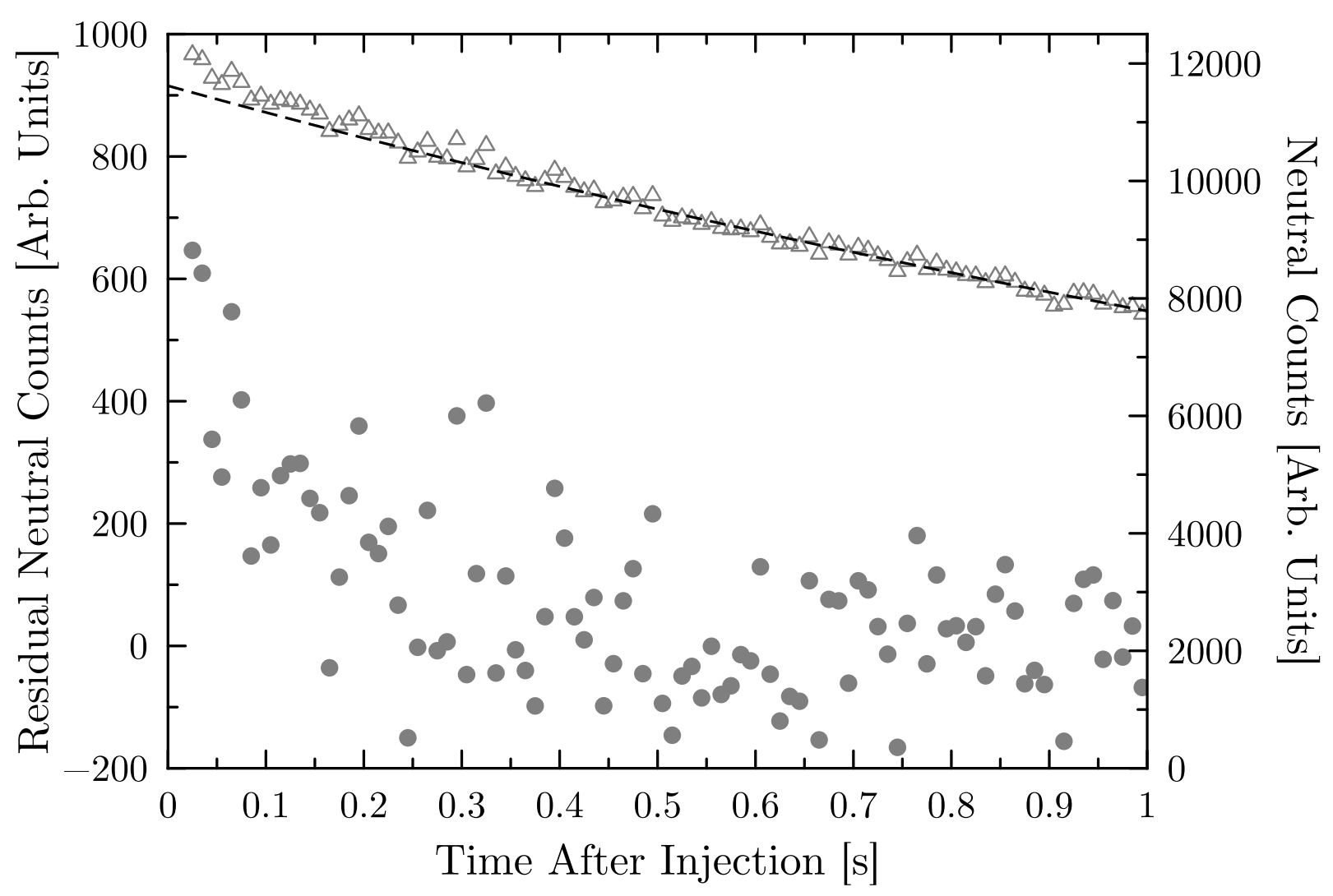

FIG. 5. Decay curve and residual signal of the $\mathrm{Pt}^{-}$beam as a function of time at a Ti:Sapphire wavelength of $750 \mathrm{~nm}$. The right vertical axis shows the original neutral counts of the decay curve $(\triangle)$ along with the exponential function fitted to the $15 \mathrm{~s}$ measurement window data (dashed line), while the left vertical axis shows the residual neutral counts after subtraction of the long-decay component corresponding to decay from the $5 d^{10} 6 s{ }^{2} \mathrm{~S}_{1 / 2}$ excited state $(\bullet)$.

\section{SUMMARY AND CONCLUSIONS}

In conclusion, the lifetimes of the two excited states of the $\mathrm{Pt}^{-}$anion are reported. The highest-lying bound excited state in $\mathrm{Pt}^{-}, 5 d^{10} 6 s{ }^{2} \mathrm{~S}_{1 / 2}$, was found to have an intrinsic lifetime of $2.54 \pm 0.10 \mathrm{~s}$, while only a range of $50-200 \mathrm{~ms}$ could be estimated for the intrinsic lifetime of the $5 d^{9} 6 s^{2}{ }^{2} \mathrm{D}_{3 / 2}$ excited fine-structure level. This lifetime range is consistent with available theoretical predictions for the ${ }^{2} \mathrm{D}_{3 / 2}$ state lifetime [38, 40]. Based on estimates of the ratio of E2 decay rates from the ${ }^{2} \mathrm{~S}_{1 / 2}$ state, the estimated lifetime of the ${ }^{2} \mathrm{~S}_{1 / 2}$ state via the $5 d^{10} 6 s{ }^{2} \mathrm{~S}_{1 / 2} \rightarrow 5 d^{9} 6 s^{2}{ }^{2} \mathrm{D}_{3 / 2}$ E2 transition is on the order of 100 days. Therefore, the measured lifetime of the $5 d^{10} 6 s{ }^{2} \mathrm{~S}_{1 / 2}$ excited state directly yields the $5 d^{10} 6 s{ }^{2} \mathrm{~S}_{1 / 2} \rightarrow 5 d^{9} 6 s^{2}$ ${ }^{2} \mathrm{D}_{5 / 2}$ transition rate. 
To date, this is the first measurement of a lifetime of a bound excited state of an atomic negative ion with a different electron configuration than that of the ground state. The present results may thus be used to benchmark ab initio calculations that include electron correlation effects, which can be expected to differ significantly between bound anionic states of different electron configurations. This is in contrast to the situation involving transitions between fine-structure levels of the same $L S$ term and electron configuration, in which the M1 transition line strength is independent of the radial part of the wavefunction and only depends on the $L, S$, and $J$ quantum numbers within the $L S$-coupling approximation (a good approximation for lighter negative ions).

Refinement of the experimental methods used in this study can be fruitfully applied to studies of other atomic negative ions with bound excited states. This includes the palladium anion $\mathrm{Pd}^{-}$, which is the final stable Group 10 negative ion yet to be studied in terms of excited state lifetimes, and the Lanthanide negative ions, several of which have been predicted to have bound excited states with lifetimes on the order of tens of seconds or longer [44].

\section{ACKNOWLEDGMENTS}

This work was supported by the Swedish Research Council (Contracts No. 821-20131642, No. 621-2015-04990, No. 621-2014-4501, and No. 621-2013-4084) and by the Knut and Alice Wallenberg Foundation. We acknowledge support from the COST Action No. CM1204 XUV/X-ray light and fast ions for ultrafast chemistry (XLIC). M.K. acknowledges financial support from the Mobility Plus Program (Project No. 1302/MOB/IV/2015/0) funded by the Polish Ministry of Science and Higher Education. We thank J. Grumer and T. Brage (Lund University) for helpful comments on theoretical aspects of the manuscript.

[1] J. R. Peterson, Y. K. Bae, and D. L. Huestis, Phys. Rev. Lett. 55, 692 (1985).

[2] T. Andersen, Phys. Rep. 394, 157 (2004).

[3] H. Hotop and W. C. Lineberger, J. Phys. Chem. Ref. Data 14, 731 (1985).

[4] D. J. Pegg, Rep. Prog. Phys. 67, 857 (2004).

[5] M. Scheer, C. A. Brodie, R. C. Bilodeau, and H. K. Haugen, Phys. Rev. A 58, 2051 (1998). 
[6] S. A. Goudsmit and T. You-Wu, Astrophys. J. 80, 154 (1934).

[7] R. Wildt, Astrophys. J. 89, 295 (1937).

[8] R. Wildt and S. Chandrasekhar, Astrophys. J. 100, 87 (1944).

[9] H. S. W. Massey and D. R. Bates, Astrophys. J. 91, 202 (1940).

[10] B. Warner, Mon. Not. R. Astron. Soc. 137, 119 (1967).

[11] P. A. Strittmatter and D. T. Wickramasinghe, Mon. Not. R. Astron. Soc. 152, 47 (1971).

[12] P. J. Sarre, Mon. Not. R. Astron. Soc. 313, L14 (2000).

[13] L. K. Fifield, Rep. Prog. Phys. 62, 1223 (1999).

[14] C. W. Walter, N. D. Gibson, D. J. Matyas, C. Crocker, K. A. Dungan, B. R. Matola, and J. Rohlén, Phys. Rev. Lett. 113, 063001 (2014).

[15] C. W. Walter, N. D. Gibson, Y.-G. Li, D. J. Matyas, R. M. Alton, S. E. Lou, R. L. Field, D. Hanstorp, L. Pan, and D. R. Beck, Phys. Rev. A 84, 032514 (2011).

[16] R. C. Bilodeau and H. K. Haugen, Phys. Rev. Lett. 85, 534 (2000).

[17] A. Kellerbauer, A. Fischer, and U. Warring, Phys. Rev. A 89, 043430 (2014).

[18] A. Kellerbauer and J. Walz, New J. Phys. 8, 45 (2006).

[19] P. Balling, L. H. Andersen, T. Andersen, H. K. Haugen, P. Hvelplund, and K. Taulbjerg, Phys. Rev. Lett. 69, 1042 (1992).

[20] H. K. Haugen, L. H. Andersen, T. Andersen, P. Balling, N. Hertel, P. Hvelplund, and S. P. Møller, Phys. Rev. A 46, R1 (1992).

[21] T. Andersen, L. H. Andersen, P. Balling, H. K. Haugen, P. Hvelplund, W. W. Smith, and K. Taulbjerg, Phys. Rev. A 47, 890 (1993).

[22] S. P. Møller, Nucl. Instrum. Methods Phys. Res. A 394, 281 (1997).

[23] D. Zajfman, O. Heber, L. Vejby-Christensen, I. Ben-Itzhak, M. L. Rappaport, R. Fishman, and M. Dahan, Phys. Rev. A 55, R1577 (1997).

[24] H. T. Schmidt, H. Cederquist, J. Jensen, and A. Fardi, Nucl. Instrum. Methods Phys. Res. B 173, 523 (2001).

[25] E. Träbert, Phys. Scr. T100, 88 (2002).

[26] A. Ellmann, P. Schef, P. Lundin, P. Royen, S. Mannervik, K. Fritioff, P. Andersson, D. Hanstorp, C. F. Fischer, F. Österdahl, D. J. Pegg, N. D. Gibson, H. Danared, and A. Källberg, Phys. Rev. Lett. 92, 253002 (2004). 
[27] P. Andersson, K. Fritioff, J. Sandström, G. Collins, D. Hanstorp, A. Ellmann, P. Schef, P. Lundin, S. Mannervik, P. Royen, K. C. Froese Fischer, F. Österdahl, D. Rostohar, D. J. Pegg, N. D. Gibson, H. Danared, and A. Källberg, Phys. Rev. A 73, 032705 (2006).

[28] J. Bernard, G. Montagne, R. Brédy, B. Terpend-Ordaciére, A. Bourgey, M. Kerleroux, L. Chen, H. T. Schmidt, H. Cederquist, and S. Martin, Rev. Sci. Instrum. 79, 075109 (2008).

[29] R. D. Thomas, H. T. Schmidt, G. Andler, M. Björkhage, M. Blom, L. Brännholm, E. Bäckström, H. Danared, S. Das, N. Haag, P. Halldén, F. Hellberg, A. I. S. Holm, H. A. B. Johansson, A. Källberg, G. Källersjö, M. Larsson, S. Leontein, L. Liljeby, P. Löfgren, B. Malm, S. Mannervik, M. Masuda, D. Misra, A. Orbán, A. Paál, P. Reinhed, K.-G. Rensfelt, S. Rosén, K. Schmidt, F. Seitz, A. Simonsson, J. Weimer, H. Zettergren, and H. Cederquist, Rev. Sci. Instrum. 82, 065112 (2011).

[30] R. von Hahn, F. Berg, K. Blaum, J. R. C. Lopez-Urrutia, F. Fellenberger, M. Froese, M. Grieser, C. Krantz, K.-U. Kühnel, M. Lange, S. Menk, F. Laux, D. A. Orlov, R. Repnow, C. D. Schröter, A. Shornikov, T. Sieber, J. Ullrich, A. Wolf, M. L. Rappaport, and D. Zajfman, Nucl. Instrum. Methods Phys. Res. B 269, 2871 (2011).

[31] Y. Nakano, W. Morimoto, T. Majima, J. Matsumoto, H. Tanuma, H. Shiromaru, and T. Azuma, J. Phys.: Conf. Ser. 388, 142027 (2012).

[32] H. T. Schmidt, R. D. Thomas, M. Gatchell, S. Rosén, P. Reinhed, P. Löfgren, L. Brännholm, M. Blom, M. Björkhage, E. Bäckström, J. D. Alexander, S. Leontein, D. Hanstorp, H. Zettergren, L. Liljeby, A. Källberg, A. Simonsson, F. Hellberg, S. Mannervik, M. Larsson, W. D. Geppert, K. G. Rensfelt, H. Danared, A. Paál, M. Masuda, P. Halldén, G. Andler, M. H. Stockett, T. Chen, G. Källersjö, J. Weimer, K. Hansen, H. Hartman, and H. Cederquist, Rev. Sci. Instrum. 84, 055115 (2013).

[33] E. Bäckström, D. Hanstorp, O. M. Hole, M. Kaminska, R. F. Nascimento, M. Blom, M. Björkhage, A. Källberg, P. Löfgren, P. Reinhed, S. Rosén, A. Simonsson, R. D. Thomas, S. Mannervik, H. T. Schmidt, and H. Cederquist, Phys. Rev. Lett. 114, 143003 (2015).

[34] Y. Enomoto, Y. Nakano, T. Masunaga, and T. Azuma, J. Phys.: Conf. Ser. 635, 022028 (2015).

[35] H. T. Schmidt, Phys. Scr. T166, 014063 (2015).

[36] M. Kamińska, V. T. Davis, O. M. Hole, R. F. Nascimento, K. C. Chartkunchand, M. Blom, M. Björkhage, A. Källberg, P. Löfgren, P. Reinhed, S. Rosén, A. Simonsson, R. D. Thomas, 
S. Mannervik, P. A. Neill, J. S. Thompson, H. T. Schmidt, H. Cederquist, and D. Hanstorp, Phys. Rev. A 93, 012512 (2016).

[37] R. C. Bilodeau, M. Scheer, H. K. Haugen, and R. L. Brooks, Phys. Rev. A 61, 012505 (1999).

[38] J. Thøgersen, M. Scheer, L. D. Steele, H. K. Haugen, and W. P. Wijesundera, Phys. Rev. Lett. 76, 2870 (1996).

[39] P. Andersson, A. O. Lindahl, D. Hanstorp, and D. J. Pegg, Phys. Rev. A 79, 022502 (2009).

[40] T. Brage and J. Grumer, arXiv:1606.08361.

[41] SNICS - Source of Negative Ions by Cesium Sputtering, National Electrostatic Corporation, USA.

[42] S. Rosén, H. T. Schmidt, P. Reinhed, D. Fischer, R. D. Thomas, H. Cederquist, L. Liljeby, L. Bagge, S. Leontein, and M. Blom, Rev. Sci. Instrum. 78, 113301 (2007).

[43] A. Corney, Atomic and Laser Spectroscopy (Oxford University Press, 1977).

[44] S. M. O’Malley and D. R. Beck, Phys. Rev. A 81, 032503 (2010). 